\title{
Quantitative analysis of IKZF1 gene deletions in pediatric B-cell precursor acute lymphoblastic leukemia: Higher levels are associated with a poorer prognosis
}

\author{
Zhizhuo Huang ${ }^{1}$, Yueping Jia ${ }^{1}$, Guorui Ruan ${ }^{1}$, Yingxi Zuo ${ }^{1}$, Jun $\mathrm{Wu}^{2}$, Aidong $\mathrm{Lu}^{1}$, yujuan \\ xue $^{1}$, Yifei Cheng ${ }^{3}$, and leiping zhang ${ }^{1}$ \\ ${ }^{1}$ Peking University People's Hospital \\ ${ }^{2}$ Peking University Shougang Hospital \\ ${ }^{3}$ Peking University Institute of Hematology
}

January 28, 2021

\begin{abstract}
Background: In pediatric B-cell precursor acute lymphoblastic leukemia (BCP-ALL), the IKZF1 gene deletions is an molecular marker of poor prognosis. We aimed to assess the prognostic effect of different levels of IKZF1 gene deletions in pediatric BCP-ALL. Procedure: IKZF1 $\triangle 2-8 /$ ALB deletions were quantified using multiplex real-time quantitative PCR (RQ-PCR) in newly diagnosed pediatric BCP-ALL patients between June 2014 and January 2018. Seventy-four patients with IKZF1 deletions of [?] $0.01 \%$ were included. Clinical characteristics, laboratory data, and treatment outcomes were analyzed. Results: The patients were divided into two groups: IKZF1 deletions of < 1\% (Group A) and of [?] 1\% (Group B). Patients in group B had a higher BCR-ABL1 positive rate than those in group A $(\mathrm{P}=0.001)$. The proportions of patients who had an age at onset of [?]10 years old, and white blood cell count [?] $50 \times 109 / \mathrm{L}$ were significantly higher in group B than in group A $(\mathrm{P}<0.05)$. The 3-year overall survival (OS) and 3-year event-free survival (EFS) rates in group B were $79 \pm 8.8 \%$ and $62.4 \pm 9.7 \%$, respectively, which were significantly lower than the 3 -year OS $(97.7 \pm 2.2 \%, \mathrm{P}=0.022)$ and 3 -year EFS $(83.2 \pm 5.8 \%, \mathrm{P}=0.019)$ in group A. Multivariate analysis revealed that the level of IKZF1 deletions of [?] 1\% and CNSL were independent risk factors of EFS. Conclusions: Pediatric BCP-ALL patients with high levels of IKZF1 gene deletions have a poorer prognosis than those with low levels.
\end{abstract}

Quantitative analysis of IKZF1 gene deletions in pediatric B-cell precursor acute lymphoblastic leukemia: Higher levels are associated with a poorer prognosis

Huang Zhizhuo ${ }^{1 \#}$, Jia Yueping ${ }^{1 \#}$, Ruan Guorui ${ }^{2}$, Zuo Yingxi ${ }^{1}$, Wu Jun $^{3}$, Lu Aidong ${ }^{1}$, Xue Yujuan ${ }^{1}$, Cheng Yifei $^{4}$, Zhang Leping ${ }^{1 *}$

${ }^{1}$ Department of pediatrics, Peking University People's Hospital, Beijing, China

2 National Clinical Research Center for Hematologic Disease, Peking University Institute of Hematology, Peking University People's Hospital, Beijing, China

${ }^{3}$ Department of pediatrics, Peking University Shougang Hospital, Beijing, China

${ }^{4}$ Department of Hematology, National Clinical Research Center for Hematologic Disease, Beijing Key Laboratory of Hematopoietic Stem Cell Transplantation, Peking University People's Hospital, Peking University Institute of Hematology, Beijing, China

\# Huang Zhizhuo and Jia Yueping contributed equally to the work. 
* Correspondence to: Dr. Zhang Leping, Department of pediatrics, Peking University People's Hospital, Beijing, China. Phone number: 0086-10-88324181. Email:zhangleping@pkuph.edu.cn

Word count (Abstract):242

Word count (Main text):2755

Number of tables: 3

Number of figures: 3

Running title: IKZF1 gene deletions in BCP-ALL

Keywords: children, acute lymphoblastic leukemia, IKZF1 gene deletion, quantitative, clinical characteristics, prognosis

\section{Abbreviations}

\begin{tabular}{ll}
\hline ALL & Acute lymphoblastic leukemia \\
\hline ALB & Albumin \\
BCP-ALL & B-cell precursor acute lymphoblastic leukemia \\
BFM & Berlin-Frankfurt-Münster \\
BM & Bone marrow \\
CNSL & Central nervous system leukemia \\
CODPL & Cyclophosphamide and prednisone/dexamethasone, vincristine, daunorubicin/idarubicin, and L-asparaginase \\
COG & Children's Oncology Group \\
CR & Complete remission \\
EFS & Event-free survival \\
FISH & Fluorescence in situ hybridization \\
HSCT & Hematopoietic stem cell transplantation \\
HR & High risk \\
IR & Intermediate risk \\
LDH & Lactate dehydrogenase \\
MICM & Morphology-immunology-cytogenetics-molecular \\
MLPA & Multiplex ligation probe-dependent amplification \\
MRD & Minimal residual disease \\
OS & Overall survival \\
RQ-PCR & Real-time quantitative polymerase chain reaction \\
SNP & Single nucleotide polymorphism \\
TKI & Tyrosine kinase inhibitor \\
UA & Uricacid \\
WBC & White blood cell \\
\hline
\end{tabular}

\section{Abstract}

Background: In pediatric B-cell precursor acute lymphoblastic leukemia (BCP-ALL), the IKZF1 gene deletions is an molecular marker of poor prognosis. We aimed to assess the prognostic effect of different levels of IKZF1 gene deletions in pediatric BCP-ALL.

Procedure: IKZF1 $\Delta 2-8 /$ ALB deletions were quantified using multiplex real-time quantitative PCR (RQPCR) in newly diagnosed pediatric BCP-ALL patients between June 2014 and January 2018. Seventy-four patients with IKZF1 deletions of [?] $0.01 \%$ were included. Clinical characteristics, laboratory data, and treatment outcomes were analyzed.

Results: The patients were divided into two groups: IKZF1 deletions of $<1 \%$ (Group A) and of [?] $1 \%$ 
(Group B). Patients in group B had a higher BCR-ABL1 positive rate than those in group A $(P=0.001)$. The proportions of patients who had an age at onset of [?]10 years old, and white blood cell count [?] $50 \times 10^{9} / \mathrm{L}$ were significantly higher in group B than in group A $(P<0.05)$. The 3 -year overall survival (OS) and 3-year event-free survival (EFS) rates in group B were $79 \pm 8.8 \%$ and $62.4 \pm 9.7 \%$, respectively, which were significantly lower than the 3-year OS $(97.7 \pm 2.2 \%, P=0.022)$ and 3 -year EFS $(83.2 \pm 5.8 \%, P=$ 0.019) in group A. Multivariate analysis revealed that the level of IKZF1 deletions of [?] $1 \%$ and CNSL were independent risk factors of EFS.

Conclusions: Pediatric BCP-ALL patients with high levels of IKZF1 gene deletions have a poorer prognosis than those with low levels.

\section{Introduction}

Acute lymphoblastic leukemia (ALL) is the most common childhood malignant cancer, with B-cell precursor ALL (BCP-ALL) accounting for approximately $80 \%$ of pediatric ALL cases. The application of molecular biological and cytogenetic techniques has revealed that the translocations $\mathrm{t}(9 ; 22) /(\mathrm{BCR}-\mathrm{ABL} 1)$, $\mathrm{t}(12 ; 21) /(\mathrm{ETV} 6 / \mathrm{RUNX} 1), \mathrm{t}(1 ; 19) /(\mathrm{E} 2 \mathrm{~A} / \mathrm{PBX} 1)$, rearrangements of the MLL gene, and hypodiploid or hypodiploid karyotypes in certain patients with ALL are associated with different prognoses. Patients benefit from risk-adapted treatment protocols. Because of progress in chemotherapy regimens and the development of risk-adapted treatment protocols, the prognosis of ALL in children has significantly improved. The 5-year event-free survival (EFS) rate of pediatric ALL from developed countries can exceed $80 \%{ }^{[1]}$, but the remaining patients are still refractory/relapsed with poor prognosis. Therefore, studies are currently focused on identifying high-risk ALL children in an early stage and carrying out the appropriate risk-adapted treatment in order to improve their prognosis.

The IKZF1 gene is located on chromosome 7p12, and encodes the lymphoid transcription protein Ikaros, which plays a key regulatory role in lymphopoiesis ${ }^{[2]}$. The deletion or mutation of IKZF1 gene are the hallmark of both BCR-ABL1 positive ALL and Ph-like ALL patients. In the most cases, IKZF1 gene deletion has been recognized as a poor prognostic factor in pediatric ALL ${ }^{[3]}$. Most data on IKZF1 deletions in pediatric ALL have been obtained through multiplex ligation probe-dependent amplification (MLPA) analyses, single nucleotide polymorphism (SNP) arrays, or polymerase chain reaction (PCR) assays, which cannot be used to detect minimal residual disease (MRD) in ALL. Recently, the levels of the IKZF1 $\Delta 2$ 8/albumin (ALB) gene deletion have been detected using a real-time quantitative polymerase chain reaction (RQ-PCR) approach and used to monitor MRD in ALL. In this study, a quantitative analysis of IKZF1 gene deletion was conducted in pediatric patients with BCP-ALL to explore the relationship between different levels of IKZF1 gene deletions and prognosis.

\section{Materials and methods}

\section{Patients}

Seventy-four newly diagnosed pediatric BCP-ALL patients (aged $<18$ years) with IKZF1 gene deletions, who underwent initial treatment in our institution between June 2014 and January 2018 were included in this study. All patients met the morphology-immunology-cytogenetics-molecular (MICM) criteria ${ }^{[4]}$. The level of IKZF1 $\Delta 2-8 /$ ALB deletions in bone marrow (BM) samples were detected by multiplex real-time quantitative PCR (RQ-PCR), as described in a previous study ${ }^{[5]}$. A patient with a IKZF1 deletion was defined as having IKZF1 $\Delta 2-8 /$ ALB deletions of [?] $0.01 \%$ of the BM sample at diagnosis. Clinical characteristics, laboratory data, and treatment outcomes were analyzed. The study was approved by the Ethics Committee of Peking University People's Hospital, and the guardians of all patients provided written informed consent.

\section{Treatment}

Initially, all patients received the induction therapy referred to as an improved ALL-Berlin-FrankfurtMünster (BFM) protocol, as described earlier ${ }^{[6,7]}$. Briefly, the patients received a CODPL induction (cyclophosphamide and prednisone or dexamethasone, vincristine, daunorubicin/idarubicin, and L-asparaginase) 
followed by consolidation and maintenance therapy. Two courses of 4 -week re-induction therapy were administered every 6 months during consolidation treatment. The consolidation treatment consisted of 15 courses of high-dose methotrexate with or without pegaspargase, 3 courses of high-dose cytarabine, 2 courses of 4 -week re-induction therapy and a course of ifosfamide. Moreover, the patients received 22-25 rounds of methotrexate, cytarabine, and dexamethasone intrathecal therapy to prevent central nervous system leukemia (CNSL). The maintenance therapy consisted of oral 6-mercaptopurine daily and weekly intramuscular methotrexate administration. The whole treatment course lasted 3-3.5 years.

The patients who were BCR-ABL1 positive were stratified in high risk (HR) group and others were stratified in intermediate risk (IR) group when they began the induction therapy. Then risk stratification was further refined by MRD detection of BM samples at the 15th or 33rd day of induction chemotherapy ${ }^{[8]}$. IR patients with MRD [?] 25\% blast at the 15th day or MRD [?] 1\% blast at the 33rd day would be upstaged to HR group. The dose and intensity of anthracyclines and high-dose methotrexate differed by risk group.

Fourteen patients underwent allogeneic hematopoietic stem cell transplantation (allo-HSCT) after several courses of consolidation treatment. Ten patients received allo-HSCT after a first complete remission (CR1), and four relapse patients chose allo-HSCT after achieving a second complete remission (CR2).

In addition to routine chemotherapy, the patients who were BCR-ABL1 positive also received a tyrosine kinase inhibitor (TKI) orally throughout the course, and some of the refractory/relapsed patients received TKIs irregularly. TKIs included the first-generation TKI imatinib mesylate (Novartis, Basel, Switzerland) (initial dose of $260-340 \mathrm{mg} / \mathrm{m}^{2} / \mathrm{d}$ ) and a second-generation TKI dasatinib (Bristol-Myers Squibb Company, Mount Vernon, USA) (initial dose of $50 \mathrm{mg} / \mathrm{m}^{2} / \mathrm{d}$ ).

MRD detection

MRD detection was performed by real-time quantitative PCR, and some common fusion transcripts, such as ETV6/RUNX1, E2A/PBX1, and BCR-ABL1, were assessed using reverse transcription PCR. Karyotyping screening was performed using a conventional karyotyping (G-banding) analysis or fluorescence in situ hybridization (FISH). Monitoring of BM samples for MRD, common fusion transcripts and IKZF1 $\Delta 2-8 /$ ALB deletions monitoring of BM sample was carried out every 2-3 months during the first 3 years after diagnosis. After which these criteria were assessed every 6 months for 2 years.

\section{Statistical analysis}

Overall survival (OS) was defined as the time from diagnosis to the date of last follow-up or death from any cause. EFS was defined as the time from diagnosis to the first event (remission failure, relapse, death, or secondary malignancy) or the last follow-up. Continuous variables were expressed as medians and ranges, while categorical variables were expressed as frequencies and percentages. The differences between different groups were analyzed using a Mann-Whitney U-test or a Chi-square test for continuous and categorical variables, respectively. OS and EFS were analyzed using the Kaplan-Meier method and a log-rank test. Cox regression analysis was performed using a multivariate analysis. Statistical analysis was performed using SPSS software (version 22.0; IBM, Armonk, NY). A $P$ value of $<0.05$ was considered statistically significant.

\section{Results}

\section{Patient Characteristics}

The median age of the 74 patients ( 46 boys and 28 girls) with IKZF1 $\Delta 2-8 /$ ALB deletions was 8 years (range, 1-17 years). The last follow-up was July 1, 2020, and the median follow-up period was 38 months (range, 2-72 months). The clinical and laboratory characteristics of the patients are summarized in Table 1.

The distribution of IKZF1 $\Delta 2-8 /$ ALB gene deletion levels

The distribution of IKZF1 deletion levels is shown in Table 2. The level of IKZF1 deletions in the 74 patients was ranged $0.01 \%$ to $243.76 \%$. There were 45 patients $(60.8 \%)$ who had IKZF1 $\Delta 2-8 /$ ALB deletions 
of $; 1 \%$, including only a single patient $(2.2 \%)$ who was BCR-ABL1 fusion gene positive, while the others were negative. Of the 29 patients with IKZF1 $\Delta 2-8 /$ ALB deletions of [?] $1 \%$, nine patients (31.0\%) were BCR-ABL1 fusion gene-positive. Patients with IKZF1 $\Delta 2-8 /$ ALB deletions of [?] $1 \%$ had a higher BCRABL1 positive rate than those with IKZF1 $\Delta 2-8 /$ ALB deletions of ; $1 \%(31.0 \%$ vs. $2.2 \%, P=0.001)$, as shown in Figure 1.

IKZF1 $\Delta 2-8 /$ ALB gene deletions and clinical features

The patients were divided into two groups: IKZF1 $\Delta 2-8 /$ ALB deletions of $<1 \%$ (Group A) and of [?] $1 \%$ (Group B). The rates of age at onset of [?] 10 years old, white blood cell count (WBC) of [?] $50 \times 10^{9} / \mathrm{L}$ at initial diagnosis, and HR patients in Group B, were significantly higher than in Group A $(P<0.05)$. Patients who were positive for the ETV6/RUNX1 fusion gene in Group A accounted for 17.8\%, which was higher than in Group B. However, the level of IKZF1 deletions was not associated with sex, WBC counts, lactate dehydrogenase (LDH), uricacid (UA), immunologic subtype, or karyotype. The central nervous system leukemia (CNSL) rate between the two groups were similar. More patients were treated with TKIs and underwent HSCT in Group B than in Group A $(P<0.05)$. The characteristics of patients with BCP-ALL with different IKZF1 $\Delta 2$-8/ALB deletion levels are summarized in Table 1.

IKZF1 $\Delta 2-8 /$ ALB gene deletions and MRD

There were no statistically significant differences between the two groups in the level of MRD at the 15th or 33rd day of induction chemotherapy or in the incidence of CNSL. In Group B, 34.5\% of the patients were refractory/relapsed, which was significantly higher than Group A ( $34.5 \%$ vs. $13.3 \%, P=0.031)$. The mortality in Group B was significantly higher than Group A $(17.2 \%$ vs. $2.2 \%, P=0.02)$, as shown in Table 1.

OS, EFS and IKZF1 $\Delta 2-8 /$ ALB gene deletions

The 3-year OS and 3-year EFS in Group B were $79.3 \pm 8.8 \%$ and $62.4 \pm 9.7 \%$, respectively, and these were significantly lower than the 3-year OS $(97.7 \pm 2.2 \%, P=0.022)$ and 3 -year EFS $(83.2 \pm 5.8 \%, P=0.019)$ in Group A (Fig. 2). The 3-year OS and 3-year EFS of Ph-negative patients in Group B were also lower than the 3-year OS $(97.6 \pm 2.2 \%$ vs. $84.7 \pm 8.1 \%, P=0.014)$ and 3 -year EFS $(85.2 \pm 5.6 \%$ vs. $59.0 \pm 12.9 \%, P$ $=0.013$ ) of Ph-negative patients in Group A (Fig. 3). Multivariate analysis revealed that the level of IKZF1 deletions of [?] $1 \%$ and CNSL were independent risk factors of EFS. While the factors such as age, gender, WBC count, CNSL, BCR-ABL1 fusion gene, risk stratification and the level of IKZF1 deletions were not associated with OS (Table 3).

\section{Discussion}

IKZF1 is composed of eight exons and encodes the transcription factor Ikaros ${ }^{[9]}$. IKZF1 alterations can occur frequently in germline cells and somatic cells, and it has been studied extensively. IKZF1 alterations in somatic cells are found in approximately $15 \%$ of pediatric patients with ALL ${ }^{[9]}$ patients, but the alternations increase in prevalence among adult patients with ALL. The most common types of IKZF1 alterations are referred to as focal deletions, such as the whole gene and exons 4-7 deletions, resulting in loss-of-function in Ikaros, whereas IKZF1 mutations were less reported, including missense mutations, nonsense mutations, and frameshift mutations ${ }^{[2]}$. IKZF1 deletions are important molecular genetic makers in the development and relapse in ALL and attract significant enough attention, until Mullighan et al. first described the IKZF1 deletions in patients with BCR-ABL1 positive ALL ${ }^{[12]}$ in 2008. Overall, the prevalence of IKZF1 deletions is $10 \%-30 \%$ in pediatric patients with B-ALL who are BCR-ABL1 negative ${ }^{[10]}$, and can be as high as $56.8 \%$ $70.6 \%$ and $27 \%-68 \%$ in pediatric patient with B-ALL who are BCR-ABL1 positive or who have Ph-like ALL. These patients are often associated with high WBC counts, older age, poor chemotherapy responses, higher risks of relapse, and insensitivity to TKIs ${ }^{[13,14]}$. In contrast, among ALL subtypes with good prognosis, the prevalence of IKZF1 deletions is low, accounting for 3\%-6\% and 15\% of ETV6-RUNX1 and hyperdiploid cases $^{[10,15,16]}$. Thus, for a long time, IKZF1 gene deletions have been established to be an independent adverse prognostic factor for patients with ALL ${ }^{[3,17]}$. Therefore, screening for IKZF1 deletions is very 
important in recognizing high-risk patients and initiating risk-adapted chemotherapy for pediatric patients with ALL.

Most of the published data on IKZF1 deletions have been generated using MLPA analyses, SNP arrays, or multiplex PCR assays. Previous results have shown that RQ-PCR based on ALB as a control gene is a reliable and sensitive method for detecting IKZF1 deletions ${ }^{[5]}$, and there is a good correlation between quantification of MRD and the level of IKZF1 deletions ${ }^{[5,17,18]}$. In this study, we conducted a quantitative analysis of IKZF1 deletions in pediatric patients with BCP-ALL to assess the impact of different levels of IKZF1 deletions on prognosis. The level of IKZF1 deletions in our patients ranged from 0.01 to $243.76 \%$, with $60.8 \%$ of them being in the IKZF1 deletions of $<1 \%$ group. The rate of IKZF1 deletions in patients with ALL who were ETV6-RUNX1 positive or hyperdiploid were account for $10.8 \%$ and $14.9 \%$, respectively, which is consistent with previous observations. Overall, $13.5 \%$ of the patients were BCR-ABL1 positive but patients with IKZF1 deletions of [?] 1\% had a significantly higher BCR-ABL1 positive rate (31.0\%) than those with IKZF1 deletions of $<1 \%(2.2 \%)$. In contrast, all patients who were ETV6/RUNX1 positive had IKZF1 deletions of $<1 \%$. Wu et al. ${ }^{[5]}$ have reported that $90 \%$ of adult patients with IKZF1 deletions had levels of IKZF1 deletions of [?] 1\%, and the rate of IKZF1 deletions in patients who were BCR-ABL1 positive was $68.8 \%$. These results differ from ours. One reason for this may be that patients who are in the high-risk group and BCR-ABL1 positive are more likely to had high levels of IKZF1 deletions; therefore, in our study fewer pediatric patients with ALL were in the high-risk group and were BCR-ABL1 positive than adult patients.

In our study, the percentages of patients whose age at onset was [?]10 years old, had WBC [?] 50x10 $10^{9} \mathrm{~L}$ at initial diagnosis, and in high-risk group were significantly higher in the IKZF1 deletions of [?]1\% group than in the IKZF1 deletions of $<1 \%$ group. In contrast, there were no differences in other clinical and laboratory characteristics between the two groups. Various clinical trials have shown that IKZF1 deletions are associated with an older age at diagnosis, a higher WBC count, higher levels of MRD after induction, and a higher risk of relapse, which corresponded with our study ${ }^{[2,13,15,20-22]}$. We found no difference in MRD at 15 and 33 days between the two groups of patients with different levels of IKZF1 deletions, whereas the rate of relapse/refractory in IKZF1 deletions of [?]1\% group was significantly higher than in the IKZF1 deletions of $<1 \%$ group, suggesting that there was more HR ALL in the IKZF1 deletions of [?]1\% group.

Almost all clinical studies have demonstrated that IKZF1 deletions are an independent adverse prognostic factor in both pediatric patients with BCP-ALL who are either BCR-ABL1 positive or negative, especially in combination with an early MRD response. In children with BCR-ABL1 negative ALL who had been treated using the BFM-2000 protocol ${ }^{[15]}$, the 5 -year EFS was $69 \%$ for patients with IKZF1 alterations vs. $85 \%$ for patients without the IKZF1 alterations. Patients with IKZF1 alterations also had a higher incidence of relapse (21\% vs. 10\%). Similar poor outcomes in pediatric patients with IKZF1 deletions were observed in a Japanese pediatric Ph-negative B-ALL cohort ${ }^{[23]}$, the AEIOP-BFM 2000 cohort $^{[24]}$, and the IC-BFM-2002 cohort $^{[25]}$. Of note, the ETV6-RUNX1 subtype should be ruled out in patients with Ph-negative B-ALL who also have IKZF1 alterations because they have favorable outcomes despite the presence of IKZF1 alterations [26]. Therefore, the prognosis is still poor for BCR-ABL1-positive and Ph-like patients who have IKZF1 deletions, even if they are treated with TKIs. In AALL0622, a Children's Oncology Group (COG) phase II trial, patients with ALL who were $\mathrm{Ph}+$ and also had IKZF1 deletions were treated with dasatinib plus intensive chemotherapy and were found to have inferior 5-year EFS and OS compared to those patients with ALL who were Ph+ but had wild type IKZF1 $(52 \%$ vs. $82 \%, P=0.04$ and $80 \%$ vs. $100 \%, P=$ 0.04 , respectively) ${ }^{[27]}$. Our results also showed that the 3-year OS and 3-year EFS in the IKZF1 deletions of [?]1\% group were both significantly poorer than the 3 -year OS $(79.3 \%$ vs. $97.7 \%, P=0.022)$ and 3 -year EFS $(62.4 \%$ vs. $83.2 \%, P=0.019)$ in the IKZF1 deletions of $<1 \%$ group. Similar results were found in BCRABL1 negative patients in corresponding different levels of IKZF1 deletions group. Therefore, the prognosis is worse in patients with higher percentage of IKZF1 deletions, regardless of BCR-ABL1 positive or negative. Because there was only one BCR-ABL1 positive patient in the IKZF1 deletions of $<1 \%$ group, we could not compare the effect of different levels of IKZF1 deletions on the prognosis of BCR-ABL1 positive children. Multivariate analysis showed that the level of IKZF1 deletions of [?] $1 \%$ and CNSL were independent risk 
factors of EFS. The level IKZF1 deletions of [?]1\% group is likely to be a poor prognosis factor of OS, although no significant statistical difference was indicated due to a limited sample size. In the future, studies on the effect of different levels of IKZF1 deletions on prognosis with large cohorts of pediatric patients with BCP-ALL should be carried out. In addition, pediatric BCP-ALL patients with the level IKZF1 deletions of [?]1\% may be stratified into high-risk groups due to poor prognosis and receive intensive chemotherapy.

Our study has several limitations. First, this was a single-center study with a small sample size. Second, due to the limitation of sample size, comparison between subgroups could not be performed.

In conclusion, pediatric BCP-ALL patients with IKZF1 $\Delta 2-8 /$ ALB deletions of $<1 \%$ had a good prognosis, whereas patients with IKZF1 $\Delta 2-8 /$ ALB deletions of [?]1\% had a poor outcomes, and these patients always had other risks such as older age, higher WBC count, with positive for the BCR/ABL1 fusion gene, and a higher relapse rate.

Conflicts of interest : The authors have no conflicts of interest to declare.

\section{References}

[1] Pui C H, Pei D, Campana D, et al. A revised definition for cure of childhood acute lymphoblastic leukemia. Leukemia, 2014,28(12):2336-2343.

[2] Linda Olsson B J. Ikaros and leukaemia. Br J Haematol, 2015,169(4):479-491.

[3] Stanulla M, Cave H, Moorman A V. IKZF1 deletions in pediatric acute lymphoblastic leukemia: still a poor prognostic marker?. Blood, 2020,135(4):252-260.

[4] Cui L, Li Z G, Chai Y H, et al. Outcome of children with newly diagnosed acute lymphoblastic leukemia treated with CCLG-ALL 2008: The first nation-wide prospective multicenter study in China. Am J Hematol, 2018,93(7):913-920.

[5] Li-Xin Wu J Z Q Y. High frequency of IKZF1 deletions in Chinese adult patients with acute lymphoblastic leukemia detected by multiplex realtime quantitative PCR. Clinical and Diagnostic Pathology, 2017,1(2):1-7.

[6] Wang Y, Zeng H M, Zhang L P. ETV6/RUNX1-positive childhood acute lymphoblastic leukemia in China: excellent prognosis with improved BFM protocol. Ital J Pediatr, 2018,44(1):94.

[7] Xue Y J, Cheng Y F, Lu A D, et al. Allogeneic Hematopoietic Stem Cell Transplantation, Especially Haploidentical, May Improve Long-Term Survival for High-Risk Pediatric Patients with Philadelphia Chromosome-Positive Acute Lymphoblastic Leukemia in the Tyrosine Kinase Inhibitor Era. Biol Blood Marrow Transplant, 2019,25(8):1611-1620.

[8] Winick N, Devidas M, Chen S, et al. Impact of Initial CSF Findings on Outcome Among Patients With National Cancer Institute Standard- and High-Risk B-Cell Acute Lymphoblastic Leukemia: A Report From the Children's Oncology Group. J Clin Oncol, 2017,35(22):2527-2534.

[9] Payne M A. Zinc finger structure-function in Ikaros Marvin A Payne. World J Biol Chem, 2011,2(6):161166 .

[10] Mullighan C G, Su X, Zhang J, et al. Deletion of IKZF1 and prognosis in acute lymphoblastic leukemia. N Engl J Med, 2009,360(5):470-480.

[11] Iacobucci I, Mullighan C G. Genetic Basis of Acute Lymphoblastic Leukemia. J Clin Oncol, 2017,35(9):975-983.

[12] Mullighan C G, Miller C B, Radtke I, et al. BCR-ABL1 lymphoblastic leukaemia is characterized by the deletion of Ikaros. Nature, 2008,453(7191):110-114.

[13] van der Veer A, Zaliova M, Mottadelli F, et al. IKZF1 status as a prognostic feature in BCR-ABL1positive childhood ALL. Blood, 2014,123(11):1691-1698. 
[14] Mullighan C G. The molecular genetic makeup of acute lymphoblastic leukemia. Hematology Am Soc Hematol Educ Program, 2012,2012:389-396.

[15] Dorge P, Meissner B, Zimmermann M, et al. IKZF1 deletion is an independent predictor of outcome in pediatric acute lymphoblastic leukemia treated according to the ALL-BFM 2000 protocol. Haematologica, 2013,98(3):428-432.

[16] Enshaei A, Schwab C J, Konn Z J, et al. Long-term follow-up of ETV6-RUNX1 ALL reveals that NCI risk, rather than secondary genetic abnormalities, is the key risk factor. Leukemia, 2013,27(11):2256-2259.

[17] Vairy S, Tran T H. IKZF1 alterations in acute lymphoblastic leukemia: The good, the bad and the ugly. Blood Rev, 2020,44:100677.

[18] Caye A, Beldjord K, Mass-Malo K, et al. Breakpoint-specific multiplex polymerase chain reaction allows the detection of IKZF1 intragenic deletions and minimal residual disease monitoring in B-cell precursor acute lymphoblastic leukemia. Haematologica, 2013,98(4):597-601.

[19] Venn N C, van der Velden V H, de Bie M, et al. Highly sensitive MRD tests for ALL based on the IKZF1 Delta3-6 microdeletion. Leukemia, 2012,26(6):1414-1416.

[20] Olsson L, Castor A, Behrendtz M, et al. Deletions of IKZF1 and SPRED1 are associated with poor prognosis in a population-based series of pediatric B-cell precursor acute lymphoblastic leukemia diagnosed between 1992 and 2011. Leukemia, 2014,28(2):302-310.

[21] Olsson L, Ivanov O I, Noren-Nystrom U, et al. The clinical impact of IKZF1 deletions in paediatric B-cell precursor acute lymphoblastic leukaemia is independent of minimal residual disease stratification in Nordic Society for Paediatric Haematology and Oncology treatment protocols used between 1992 and 2013. Br J Haematol, 2015,170(6):847-858.

[22] van der Veer A, Waanders E, Pieters R, et al. Independent prognostic value of BCR-ABL1-like signature and IKZF1 deletion, but not high CRLF2 expression, in children with B-cell precursor ALL. Blood, 2013,122(15):2622-2629.

[23] Asai D, Imamura T, Suenobu S, et al. IKZF1 deletion is associated with a poor outcome in pediatric B-cell precursor acute lymphoblastic leukemia in Japan. Cancer Med, 2013,2(3):412-419.

[24] Palmi C, Valsecchi M G, Longinotti G, et al. What is the relevance of Ikaros gene deletions as a prognostic marker in pediatric Philadelphia-negative B-cell precursor acute lymphoblastic leukemia?. Haematologica, 2013,98(8):1226-1231.

[25] Volejnikova J, Mejstrikova E, Dorge P, et al. Ikaros (IKZF1) alterations and minimal residual disease at day 15 assessed by flow cytometry predict prognosis of childhood BCR/ABL-negative acute lymphoblastic leukemia. Pediatr Blood Cancer, 2013,60(3):420-427.

[26] Lilljebjorn H, Henningsson R, Hyrenius-Wittsten A, et al. Identification of ETV6-RUNX1-like and DUX4-rearranged subtypes in paediatric B-cell precursor acute lymphoblastic leukaemia. Nat Commun, 2016,7:11790.

[27] Slayton W B, Schultz K R, Kairalla J A, et al. Dasatinib Plus Intensive Chemotherapy in Children, Adolescents, and Young Adults With Philadelphia Chromosome-Positive Acute Lymphoblastic Leukemia: Results of Children's Oncology Group Trial AALL0622. J Clin Oncol, 2018,36(22):2306-2314.

\section{Legends}

Figure 1 The distribution for different levels of IKZF1 deletions in patients with BCR-ABL1 positive and negative.

Figure 2 Kaplan-Meier survival curve of OS (a) and EFS (b) of the patients in IKZF1 deletions of [?]1\% group and IKZF1 deletions of $¡ 1 \%$ group. 
Figure 3 Kaplan-Meier survival curve of OS (a) and EFS (b) of BCR-ABL1 negative patients in IKZF1 deletions of [?]1\% group and IKZF1 deletions of $¡ 1 \%$ group.

\section{Hosted file}

IKZF1-Table 1.pdf available at https://authorea.com/users/392231/articles/506209quantitative-analysis-of-ikzf1-gene-deletions-in-pediatric-b-cell-precursor-acutelymphoblastic-leukemia-higher-levels-are-associated-with-a-poorer-prognosis

\section{Hosted file}

IKZF1-Table2.pdf available at https://authorea.com/users/392231/articles/506209-quantitativeanalysis-of-ikzf1-gene-deletions-in-pediatric-b-cell-precursor-acute-lymphoblasticleukemia-higher-levels-are-associated-with-a-poorer-prognosis

\section{Hosted file}

Table 3.pdf available at https://authorea.com/users/392231/articles/506209-quantitativeanalysis-of-ikzf1-gene-deletions-in-pediatric-b-cell-precursor-acute-lymphoblasticleukemia-higher-levels-are-associated-with-a-poorer-prognosis
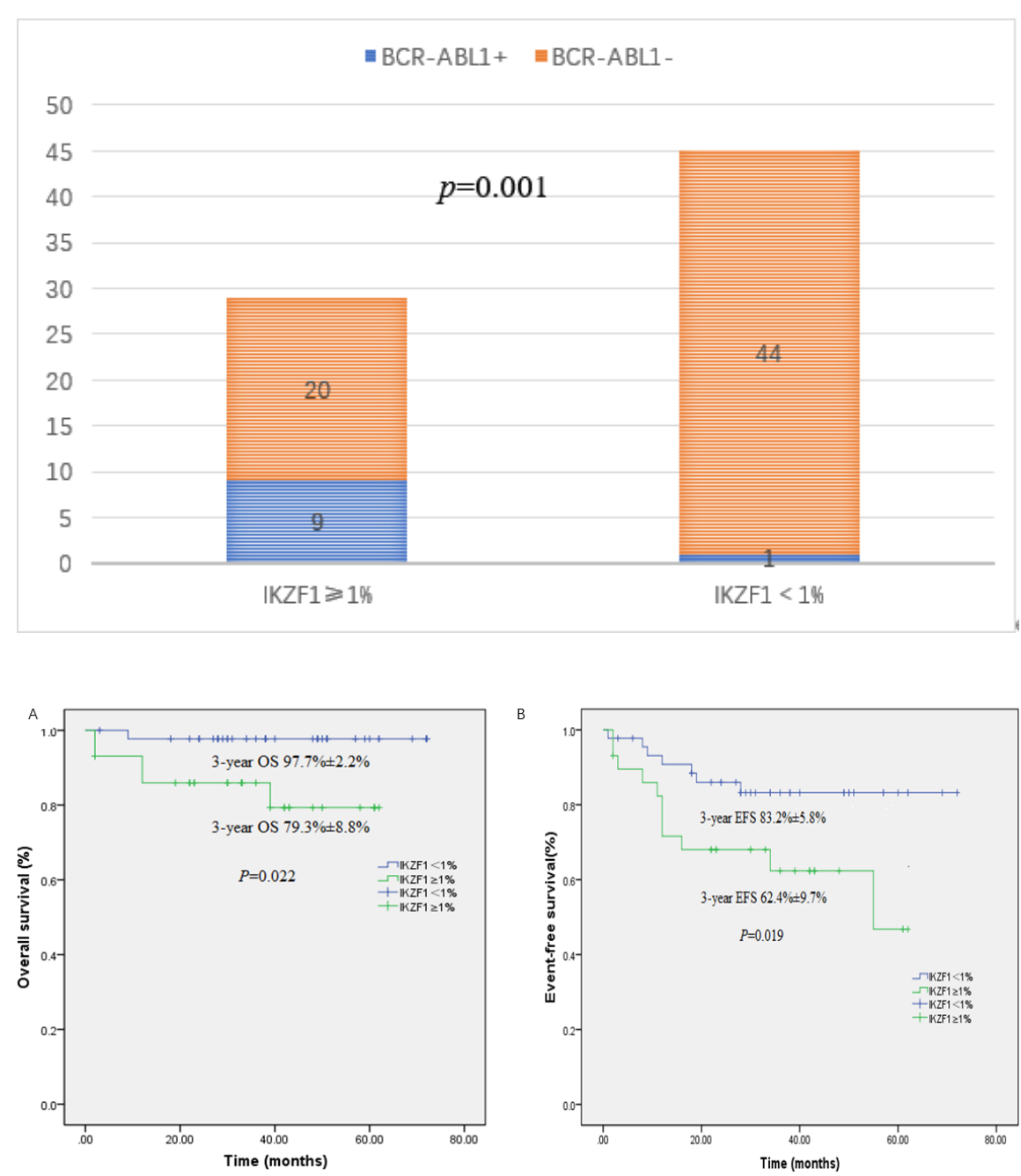
A

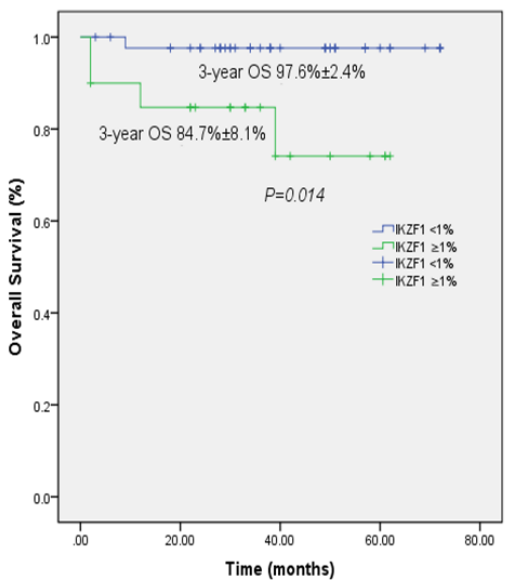

B

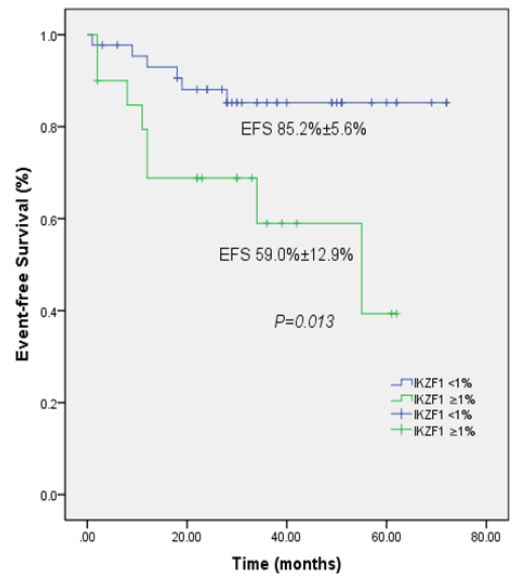

